E-Review 1-2013

Bologna (BraDypUS) 2014

\#usopubblico

ISSN: 2282-4979

ISBN: $978-88-98392-05-6$

DOI: $10.12977 /$ ereview35

pp. $259-266$

\title{
10 volti per la Liberazione. Una mostra per le strade della città
}

Nel saggio si dà conto delle vicende che hanno segnato "10 volti per la Liberazione", mostra diffusa nelle strade di Parma, allestita in occasione del 25 aprile 2013 dal Centro studi movimenti con sagome a grandezza naturale di alcuni protagonisti della lotta partigiana. L'autrice affronta i problemi incontrati nel corso dell'allestimento e le reazioni che l'iniziativa ha suscitato, elementi che investono direttamente la riflessione storiografica avendo a che vedere con l'uso pubblico della storia, con il rapporto tra memoria pubblica e privata e con il ruolo rivestito dagli storici nella costruzione della memoria collettiva.

The essay is about the events happened during the exhibit called "10 faces for Liberation", set up in 2013 in the streets of Parma during the celebration of April 25th by Centro studi movimenti and made of full-sized pictures of ten partisans, both men and women. The authors had to face some problems during the setting up of the exhibit, because it engendered many reactions and questions having straightly to do with historiography, such as public use of history, the relationship between private and public memory and the role of historians in the building of collective memory.

(Contributivideo disponibili online: 4eXcWz705aj3L-3HzduwYlfhWab).

In occasione del 25 aprile scorso, come Centro studi movimenti ci siamo gettati, non senza ostacoli e difficoltà, nell'agone della memoria resistenziale, organizzando nella città in cui lavoriamo - Parma - una mostra diffusa nelle strade e nelle piazze, costruita con le immagini stampate a grandezza naturale di alcuni protagonisti della lotta di Liberazione. Tra i tanti, ne abbiamo selezionati dieci, sette 
uomini e tre donne, con l'idea di raccontare la loro esperienza per ragionare sulla loro scelta partigiana: ripensare quelle vite, infatti, significava per noi valorizzare la capacità di scegliere da che parte stare in uno dei periodi più bui dell'Italia recente, nel quale la storia personale di ognuno si è intrecciata inevitabilmente con la storia generale, e nel quale ciascuno - uomo o donna, giovane o anziano, al di là dei propri orientamenti religiosi o politici e delle proprie condizioni sociali - ha dovuto fare i conti con se stesso.

Quelle scelte e quei volti, però, non potevano essere chiusi in una stanza, ridotti a medaglioni della retorica antifascista, abbandonati alla generosa visita degli ormai pochi interessati. Volevamo che quei volti invadessero la città, la costringessero a confrontarsi con il suo passato, volevamo che ognuno, imbattendosi in loro, se ne domandasse la ragione. Per questo li abbiamo collocati in spazi esterni, significativi per la loro vita e la loro storia: la loro casa, il loro posto di lavoro, le basi della clandestinità o i luoghi della detenzione e della tortura in cui diversi di loro incapparono.

Così è nato 10 volti per la Liberazione, un percorso facilmente percorribile nel centro storico che, ad ogni volto, evocava la città tra il settembre 1943 e l'aprile 1945; un'esperienza nuova per Parma che mirava ad investire la città e i suoi spazi prefiggendosi obiettivi di diffusione culturale anche se, in breve, ha assunto

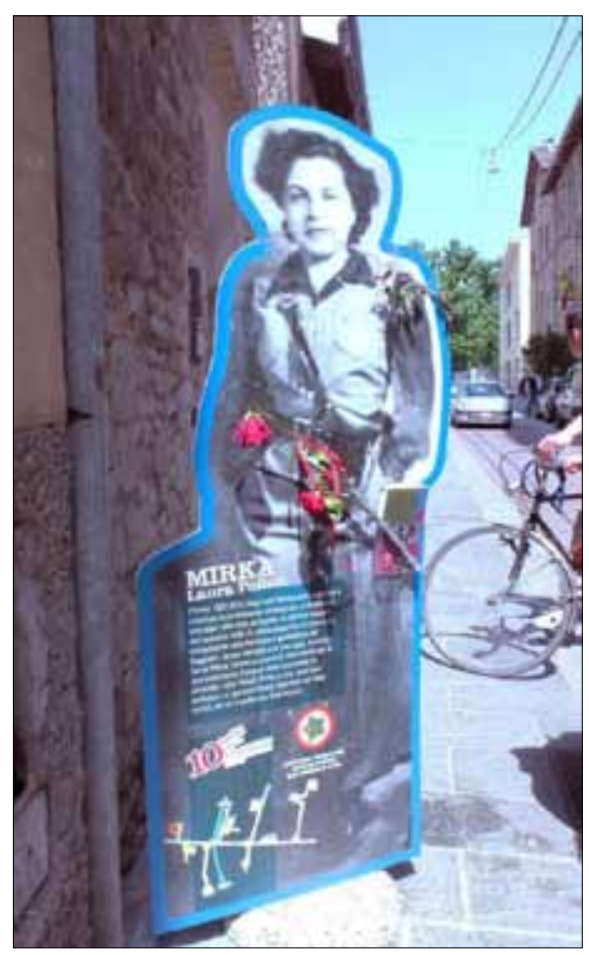

Sagoma di Laura Polizzi "Mirka” i tratti di una mobilitazione antifascista. Motivo per cui non si può parlare della mostra e delle sue ragioni storiografiche senza raccontare cosa intorno ad essa è maturato e quali problemi ha sollevato.

\section{Scelte personali}

Il primo si è presentato fin dalla fase d'ideazione ed ha riguardato la selezione dei 10 volti, avvenuta in base all'idea di proporre biografie non solo significative per la storia di Parma, ma anche capaci di mostrare aspetti diversi di quella ricca e complessa storia che è stata la lotta di Liberazione.

Ciascuno di loro, infatti, avrebbe dovuto raccontare un differente modo di opporsi all'occupazione tedesca e al collabo- 
razionismo della Rsi, ma su questo criterio non erano d'accordo alcune associazioni partigiane che, invece, spingevano e incalzavano affinché scegliessimo solo i combattenti insigniti di medaglia d'oro al valor militare. Prospettiva per noi non solo storiograficamente superata dalla mole di studi e approcci che, negli ultimi trent'anni, hanno finalmente popolato la storia della Resistenza di una miriade di volti ed esperienze, ma che avrebbe anche escluso tutte e tre le donne presenti nella mostra.

Distanti per indole e formazione scientifica da intenti celebrativi, ripensare e raccontare quelle vite per noi non significava fare un monumento all'eroismo resistenziale, quanto valorizzare le singole scelte individuali che hanno segnato il passaggio dal totalitarismo fascista alla Repubblica democratica, attraverso il recupero di quegli aspetti personali che sempre caratterizzano la vita di ogni uomo e donna, tanto nel privato quanto nella dimensione pubblica. Amori e affetti, dunque, insieme a speranze e delusioni, debolezze e qualità. Non volevamo leggende di eroi o esseri sovrumani ma racconti di uomini e donne comuni, anche perché una narrazione di questo tipo, secondo noi, avrebbe favorito un processo d'identificazione e dunque, da parte di ognuno, un ripensamento critico di sé, delle proprie scelte e del proprio ruolo in questa società'.

\section{Stratificazioni del ricordo}

Il secondo problema che si è posto, e su cui ci siamo trovati a dover riflettere pubblicamente dopo la sparizione di una sagoma già la sera prima dell'inaugurazione, è stato innescato dalle lamentele, tramite le colonne del principale giornale cittadino - "La Gazzetta di Parma" della figlia di Maria Zaccarini, partigiana "Livia", la quale si è pubblicamente detta contraria all'esposizione dell'im-

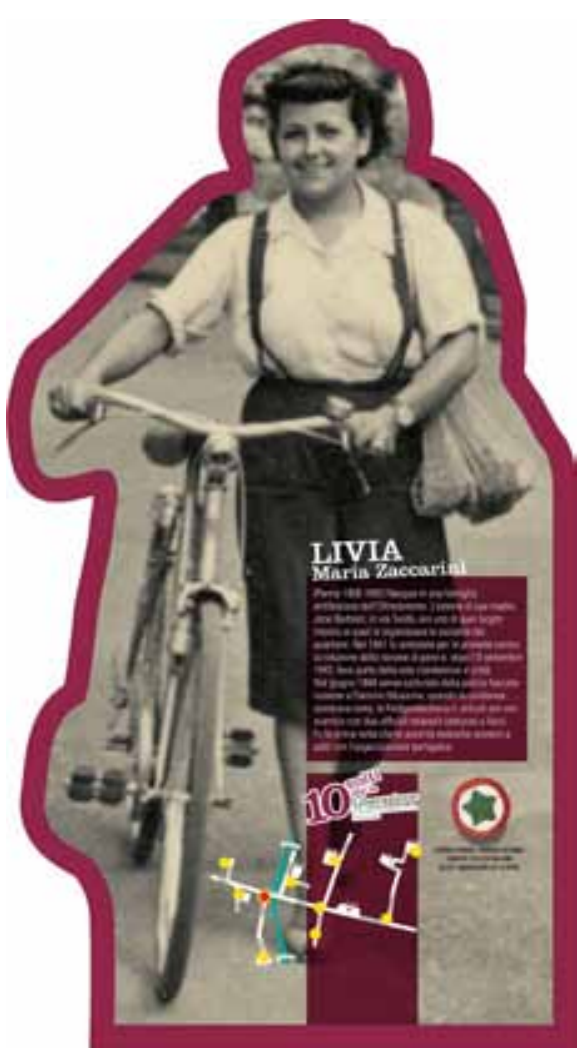

Sagoma di Maria Zaccarini "Livia" 
magine della madre, e dunque contenta della sua scomparsa. Al di là dei dubbi sulle responsabilità del furto, la presa di posizione della figlia, dura e perentoria, ci ha spinto a uscire dalle secche della polemica sui giornali per stimolare in città una discussione più alta, che avesse a che fare con la stratificazione del ricordo e dei diversi livelli di memorie che - senza essere sempre sovrapponibili - convivono nel presente. La figlia di Maria Zaccarini ha cioè rivendicato la sua memoria privata, senza dubbio densa di emozioni e sofferenza per il vuoto che la scomparsa della madre ha lasciato. Ma, accanto ad essa, esiste una memoria pubblica - civile o storiografica che sia - che, con il suo sguardo al passato, deve orientare la società, farsi promotrice d'identità collettiva e proporre valori e idealità da preservare nel presente.

Le polemiche hanno fatto dunque emergere il problema della discordanza tra queste differenti memorie che, se talvolta convergono e promuovono unitariamente una stessa memoria pubblica, altre volte - come in questo caso - divergono e entrano in conflitto. Chi negli studi storici lavora sulle biografie, per fare l'esempio più lampante, sa perfettamente quali siano le difficoltà nel relazionarsi con familiari che desiderano contribuire attivamente alla ricostruzione, condizionando e indirizzando l'interpretazione e la ricerca. Le esigenze della figlia di Maria Zaccarini di non voler ricordare pubblicamente la bellissima storia della madre si è scontrata con altre esigenze: quella di noi storici - che in quella figura ab-

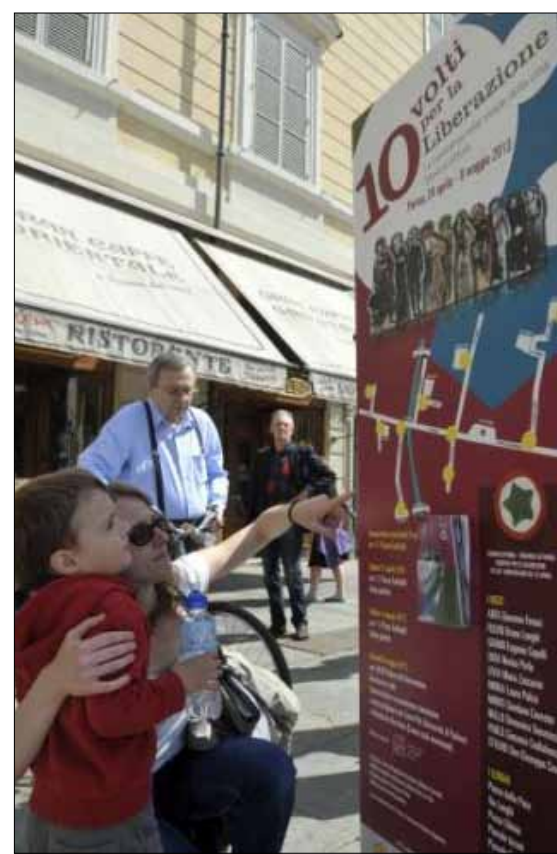

Pannello introduttivo al percorso della mostra diffusa biamo individuato un esempio significativo per riflettere sul periodo di cui è stata protagonista - e quella delle istituzioni che, tramite la vita di quell'allora giovane donna, il suo rigore morale, la sua scelta partigiana, volevano proporre alla città e alle nuove generazioni un esempio straordinario di assunzione di responsabilità in relazione all'esistente e alla sua giustizia.

\section{Oltraggio e cura}

Il terzo problema - quello che ha sollevato senza dubbio più clamore - è stato quello dei danneggiamenti che, fin dai primi giorni, uno dopo l'altro, hanno colpito quattro sagome, decapitate e imbrattate. Immaginavamo tutti i pericoli che una 
mostra di questo tipo - con installazioni esterne, ben visibili e senza alcuna protezione - avrebbe potuto correre, eppure abbiamo tenacemente lavorato al progetto perché convinti che la storia della lotta partigiana, e dei valori che essa porta con sé, non sia più trasmissibile se non in una forma pubblica e di massa, fuori dalle stanze protette delle cerimonie ufficiali o da quelle frequentate da coloro che in quei valori già si riconoscono.

Quindi, sebbene turbati da quanto accadeva, abbiamo cercato di fare di quel turbamento uno stimolo, un'occasione per riflettere e rilanciare, sia sul piano civile che storiografico, i nodi che di volta in volta si sono presentati. Anche noi, come il resto della città, ci siamo più volte chiesti se fosse stato vandalismo qualunquista o un gesto politico cosciente l'autore di quegli sfregi che, indipendentemente dalla loro origine, hanno fatto emergere tutto l'impoverimento e le contraddizioni che, anche in una città come Parma - medaglia d'oro per la Resistenza e orgogliosa della sua tradizione antifascista - lacerano la memoria collettiva proprio sul terreno dell'antifascismo e della lotta partigiana.

E su questo abbiamo tentato di chiamare la città a riflettere, esponendo cartelli al posto delle sagome trafugate e avviando dibattiti in rete e sui giornali, stimolando la discussione anche sulla grossa parte di responsabilità che - circa questo impoverimento - è per noi da ascrivere al disinteresse e all'indifferenza verso qualsivoglia politica della memoria che ha segnato - a Parma, ma certo non solo - la classe dirigente degli ultimi vent'anni. Tutta proiettata in affari e profitti, essa non ha certo cessato di celebrare formalmente la Resistenza, ma, con rievocazioni

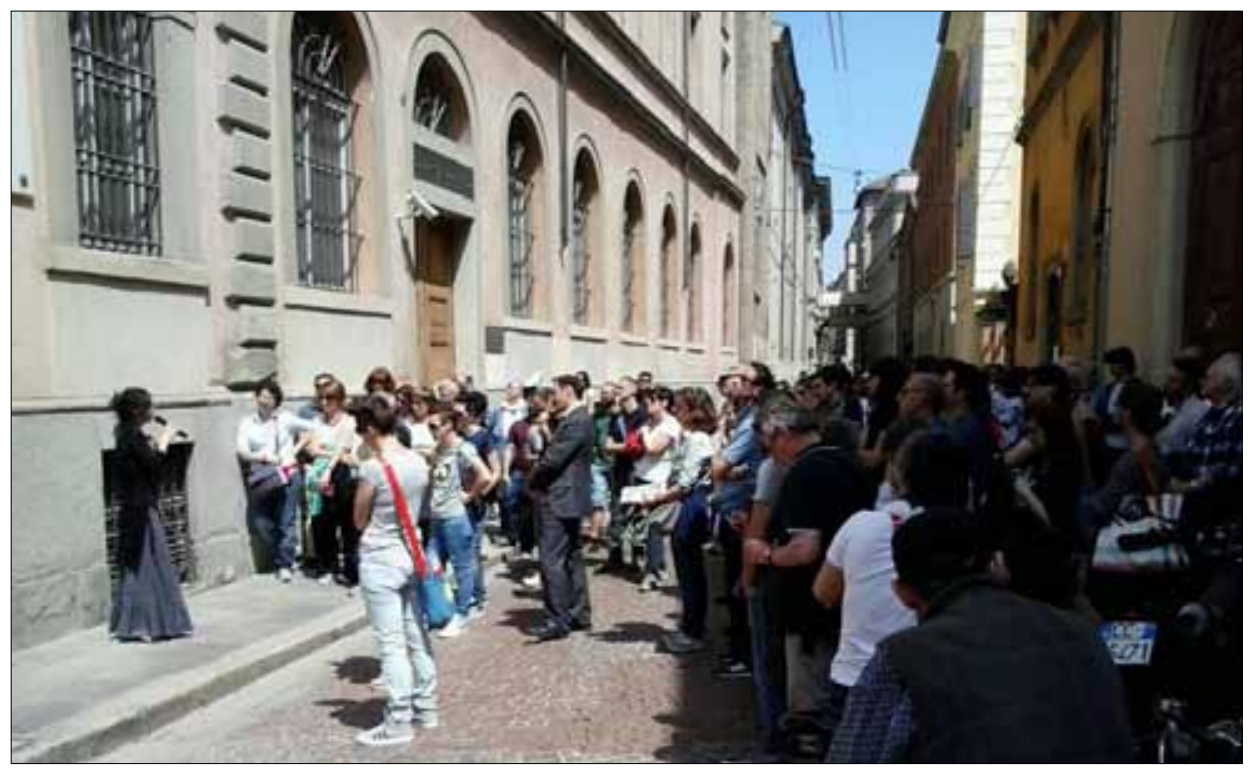

Visita guidata in via Cavestro 


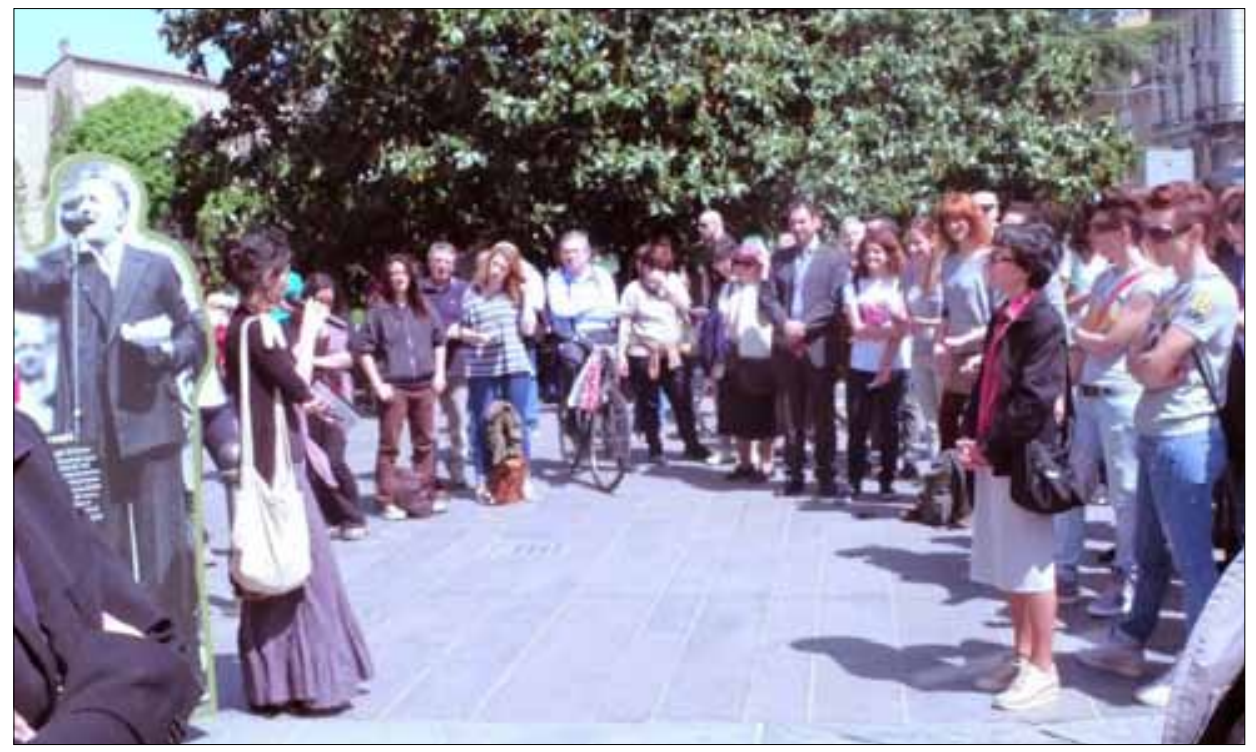

Visita guidata alla sagoma di Giacomo Ferrari “Arta”

stanche e rituali, l'ha resa - come altre volte accaduto in passato - un ossificato e inservibile monumento al tempo andato. L'antifascismo è stato trattato come un'ingombrante zavorra del Novecento, un peso del quale disfarsi per avere finalmente un paese pacificato, dove tutti quanti - rossi e neri, fascisti e antifascisti - potessero riconoscersi.

Per questo l'aggressione che la memoria dell'antifascismo e della lotta partigiana in questi anni ha subito è stata molto più sottile e pericolosa rispetto a quella dei decenni precedenti, quando era soprattutto la cultura neofascista ad avversarla. Insidiosa ed efficace, tanto che oggi è proprio con la profonda delegittimazione politica di cui l'antifascismo è vittima che dobbiamo confrontarci, soprattutto rispetto alle nuove generazioni cresciute in questo clima disinteressato al passato. Per questo abbiamo voluto esibire pubblicamente i volti partigiani, anche rischiando di esporli all'oltraggio. Significava per noi non solo chiamare i cittadini a riconoscersi nella loro scelta, ma anche indicare nuovamente alla collettività le radici della nostra convivenza.

E ciò che è accaduto durante i venti giorni della mostra, in effetti, ha reso evidente quanto, in città, discussione e presa di posizione si fossero risvegliate: semplici cittadini hanno spontaneamente iniziato a riparare le sagome danneggiate o a proteggerle durante la notte, portandosele a casa senza che nessuno glielo chiedesse, altri hanno portato loro dei fiori, altri ancora hanno cambiato l'immagine del proprio profilo facebook con quello di una delle 10 figure, coinvolgendo in questa mobilitazione di solidarietà anche uomini e donne di altre città. Studenti delle 
scuole hanno scritto al giornale cittadino per esprimere la propria indignazione e, sebbene con colpevole ritardo, anche partiti e sindacati hanno pubblicamente espresso solidarietà alla mostra; e richiamato il valore dell'antifascismo quale fondamento delle istituzioni democratiche. La risposta attiva e cosciente agli oltraggi che è maturata in persone, associazioni e istituzioni ha cioè trasformato la presa in cura delle sagome in una metaforica cura della propria storia e dei suoi valori migliori.

E così, rinfrancati dalla solidarietà crescente che la città mostrava a quei volti, abbiamo deciso di non ristampare le sagome danneggiate e decapitate, ma di enfatizzare lo scempio cui erano state sottoposte, applicando uno specchio al posto del loro viso, cosicché ognuno ci si potesse rivedere, e scegliere, a propria volta, quale posizione prendere di fronte all'oltraggio evidente che questa società troppo spesso muove alla memoria di quel passato e soprattutto ai valori che esso ha espresso. A ogni nuovo sfregio, quindi, invece di rassicurarci gridando alle provocazioni neofasciste, abbiamo rilanciato la battaglia per la cura di quella memoria, indirizzandola a tutti coloro che riconoscevano quei volti come propri. La risposta - come detto - è stata confortante ed emozionante: oltre ai singoli gesti di solidarietà, sempre più persone partecipavano e seguivano le visite guidate che ogni settimana organizzavamo e l'ultima, poi, il 4 maggio, ha dato senz'altro il segno più vigoroso: un piccolo corteo di qualche centinaio di persone che si è riappropriato della propria città e della sua storia più bella².

Le vicende della mostra hanno segnato tutti, la città, il nostro modo di fare ricerca e la mostra stessa. Per questo abbiamo deciso di modificare nel merito e nell'organizzazione anche l'ultimo incontro previsto, vale a dire la conferenza conclusiva del 9 maggio che, a Palazzo del Governatore, avrebbe dovuto riflettere con Santo Peli sulle specificità del movimento clandestino rispetto alle formazioni militari in montagna. Ormai

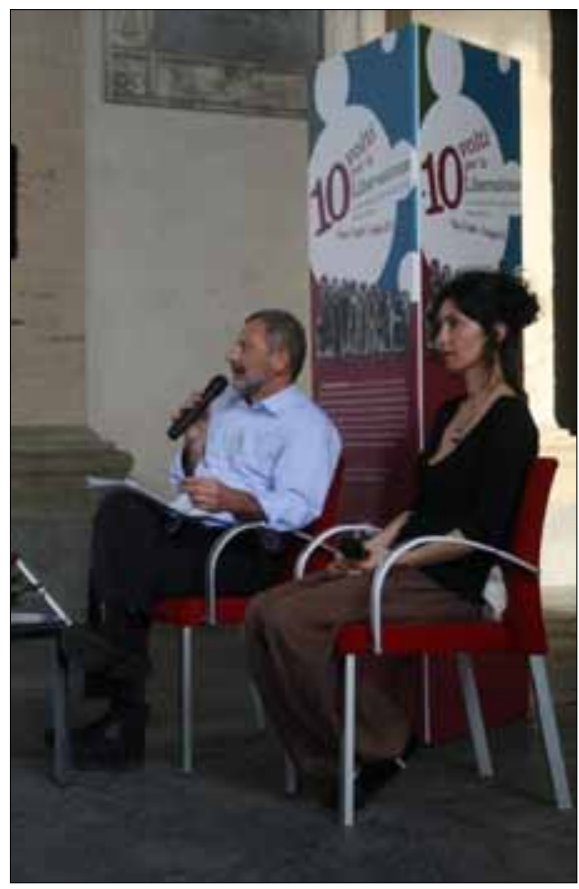

Conferenza conclusiva 
non potevamo più concludere quell'esperienza tornando nel chiuso dei palazzi, riportando quella storia collettiva nel privilegio protetto della condivisione tra pochi, ma dovevamo restituirla alla collettività, alla città nel suo insieme. Per l'assemblea finale, dunque, abbiamo voluto la piazza politica più importante per la storia di Parma, piazza Garibaldi, i Portici del palazzo municipale e i loro innumerevoli passanti, perché la memoria collettiva è un bene comune del quale non ci si può disfare, un bene comune non solo da tutelare dal logoramento del tempo ma da riconquistare alla seduzione delle sirene del potere esistente.

\section{Risorse}

Centro studi movimenti Parma http://www.csmovimenti.org

Video conferenza conclusiva con Santo Peli e Margherita Becchetti, Parma 9 maggio 2013 http://youtu.be/UkhjCGqhUnQ 